Cometary scientists soon realized, however, that they had done little more than reveal the huge extent of their ignorance. Basic quantities such as the mass and density of Comet Halley were unknown, never mind the more interesting details of its origin, original size, internal structure and surface variability. And, worse still, astronomers had no idea whether Halley is typical of comets in general or is something of an odd-ball (Halley is relied upon for the calibration and interpretation of other cometary events).

The success of the 1986 European and Japanese cometary space missions seemed to put a nail in the coffin of NASA's cometary exploration programme. Not only had NASA missed the opportunity to rendezvous with Comet Temple-2 after visiting Comet Halley, but its plans to observe Halley from the Astro-1 space platform were quashed by the Challenger space-shuttle disaster. As a final curtain, the US Congress has now cancelled the Comet Rendezvous Asteroid Flyby spacecraft, which would have orbited a comet for a year or two before finally landing on its surface.

Brandt and Chapman seem to have two dilemmas. The publisher's blurb for Rendezvous in Space suggests that our understanding of comets has changed completely since the investigation of Comet Halley. The authors, quite rightly, will have none of this, and their book stands firmly on the shoulders of their excellent Introduction to Comets (Cambridge University Press, 1981). Strong emphasis is placed on how cometary scientists have been painstakingly revealing the secrets of comets for more than two centuries. Halley is just another chapter, albeit a rather long one.

The second dilemma concerns the book's level. Unfortunately, the authors provide a better introductory guide to cometary science in their 1981 book than in this newish work. (I use the word newish advisedly because the similarity between the two books is quite marked.) New science is often more difficult to understand than old science, so to appease the intended innumerate reader, Brandt and Chapman have written the first 211 pages without resource to any equations. All the relevant mathematics has been shuffled ignominiously into a set of appendices. Here one can revel in the joys of such things as plasma physics and the cometary surface energy balance. Detailed computer programs are given for calculating cometary ephemerides and there is a collection of 11 pictures aimed at providing an overview of the in situ measurements made of the plasma close to Comet GiacobiniZinner and Comet Halley, and of the dust and gas near the latter. But the dichotomy is too marked; readers who enjoy the appendices will find the preceding text too bland, whereas those who like the text will be swamped by the appendices.

This is a good book for newcomers, but old hands will be disappointed that these first-class authors have not doubled the length of their text and been more critical in their approach to such topics as the origin of comets, the prevalence of cometary impacts, the decay of comets and the characteristic range of members of the cometary family. I would have been happier if even more emphasis had been placed on the gaping holes of ignorance that pepper cometary science.

David W. Hughes is in the Department of Physics, University of Sheffield, Sheffield S3 $7 R H$, UK.

\section{A selectionist view}

\section{Walter F. Eanes}

The Causes of Molecular Evolution. By John H. Gillespie. Oxford University Press: 1992. Pp. 336. £25, \$35.

OVER the past decade, arguments for the neutral theory of molecular evolution have enjoyed prominence through books written by Motoo Kimura, Masatoshi Nei, Wen-Hsiung Li, Dan Graur and others. Now, John Gillespie, a population geneticist, provides a selectionist's view of molecular evolution. This book represents the fusion of his long-term interest in the evidence for unequal rates of molecular evolution at the amino-acid level and in mathematical models of selection in varying environments. What emerges is an assessment of the neutralist model of molecular evolution and an elaboration of the process by which he proposes selection occurs at the aminoacid level.

The book encompasses several related topics. First, Gillespie discusses his favourite case studies of so-called protein micro-adaptations, and summarizes what he feels are the generalities about electrophoretic variation that emerged from studies in the 1970s. Next, he examines the main studies of molecular evolution at the DNA level, the data and logic behind the molecular clock, and the neutralist contention that proteins evolve at a constant rate. He includes a useful discussion of the theory of point processes, which lays the foundation for his view that substitution rates are not constant, but reflect episodic selection.

About a third of the book is devoted to mathematical models of selection in varying environments. This is a slippery topic because of the increased dimensionality, and opens a large number of problems for both the mathematician and the experimentalist. At this point, readers should be warned. This is not textbook population genetics, but diffusion theory. And it is not for the lighthearted - although discussed in a relaxed conversational style, the topic will be intractable to many. The general theory is important to Gillespie's overall theme, but presented in this much detail it becomes a distraction from the main issues. It will undoubtedly be skipped over by most readers. Nevertheless, the take-home lesson of Gillespie's models is that, in contradiction to earlier theory, selection in random environments can maintain genetic variation, and his mathematical demonstration of this capability is an important contribution. In the final chapters, Gillespie describes and assesses the neutral theory and its variants.

I like this book, and it is valuable to have many elements of the debate under one cover. I am sympathetic to Gillespie's case, although this is not to say that I agree with all his interpretations, or that he is without bias. For example, he is critical of the work on chemostat selection in Escherichia coli, which found no evidence for selection acting on electrophoretic polymorphisms, and he dismisses it because it does not live up to his experimental standards. Yet some other studies that are in my opinion of dubious value become his paradigm for selection. Gillespie's critique will not (nor should it) lead to the discarding of the neutral theory, which will continue to serve as a useful, albeit limited, null hypothesis. He nevertheless raises many important issues, such as the similarity of the patterns of allele frequency expected under both models, problems with assuming equilibrium populations and the importance of defining the spectrum of mutations.

The book is very much the gospel according to Gillespie, yet he makes many of his cases well, especially the argument for varying rates of substitution. As a proponent of the selectionist view he is not alone, and this work will begin to counter the influence that defenders of the neutral theory have enjoyed over the past decade. Given the explosion of molecular data that will emerge in the future, this is a timely contribution which will promote interest in the study of adaptation at the molecular level.

Walter F. Eanes is in the Department of Ecology and Evolution, State University of New York, Stony Brook, New York 11794, USA. 